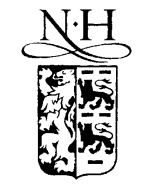

ELSEVIER

\title{
Effect of temperature on the terminal relaxation of branched polydimethysiloxane
}

\author{
C.M. Roland *, P.G. Santangelo \\ Chemistry Division, Code 6120, Naval Research Laboratory, Washington, DC 20375-5342, USA
}

\begin{abstract}
The effect of long chain branching on the rheology of polydimethylsiloxane (PDMS) was investigated. Due to the low glass temperature and high thermal stability of PDMS, the terminal relaxation could be measured at temperatures more than $270^{\circ}$ above the polymer's glass temperature. Over the measured range, the data for all samples conformed to the time-temperature superposition principle and exhibited Arrhenius behavior. Activation energies, determined from the zero-shear viscosity of a linear and a commercial PDMS were equal to within the experimental error. Branching of the latter by chemical reaction did not change the temperature dependence of its terminal shift factors. This demonstrates that similar to polyisobutylene, but quite distinct from polyethylene, 1,4-polyisoprene, and 1,4polybutadiene, long-chain branching does not affect the temperature dependence of the terminal rheology of PDMS. Published by Elsevier Science B.V.
\end{abstract}

\section{Introduction}

Among the various properties of polymers, their response to temperature changes is among the most important, both for fundamental and practical reasons. The temperature dependence of segmental relaxation is determined almost entirely by that of the local friction coefficient. Length scales beyond that associated with the segmental relaxation involve chain modes, which for unentangled polymers are described by the Rouse model [1]. Although there are discrepancies with

\footnotetext{
${ }^{*}$ Corresponding author. Tel.: +1-202 767 1719; fax: +1-202 7679594.

E-mail address: mike.roland@nrl.navy.mil (C.M. Roland).
}

some experimental results [2,3], in general the Rouse description works well. The relaxation time of the $p$ th Rouse mode is given by [4]

$\tau_{p}=\frac{l^{2} M^{2} \varsigma_{0}}{3 \pi^{2} m^{2} p^{2} k T}$,

where $l$ is the Kuhn length, $M$ and $m$ the respective chain and monomer molecular weights, and $\zeta_{0}$ the monomeric friction coefficient. Along with the explicit $T$ in the denominator and any (small) variation of $l$ with temperature, $\zeta_{0}$ is the only temperature sensitive quantity in Eq. (1). This $\zeta_{0}$ can moreover be identified with the segmental friction factor. This suggests that the Rouse and local segmental motions are governed by the same friction factor, and hence are expected to have the same temperature dependence. However, this is 
not the case, as evidenced by thermorheological complexity in the softening zone of the viscoelastic spectrum $[2,5,6]$.

The lowest frequency motion of high polymers, corresponding to the terminal zone, is most commonly described by the reptation model. For linear polymers, the terminal reptation time is related to the longest Rouse relaxation time as [4]

$\tau_{\mathrm{R}}=\frac{3 M}{M_{\mathrm{e}}} \tau_{p=1}$

where $M_{\mathrm{e}}$ is the molecular weight between entanglements. This relaxation time is related to the terminal viscosity as [4]

$\eta_{0}=G_{N} \tau_{\mathrm{R}}$

where $G_{N}$ is the plateau modulus. The presence in Eq. (2) of the local segmental friction factor, coming from the assumption of Rouse dynamics within the tube of entanglement constraints, indicates that the reptation model fails to anticipate the difference in temperature dependence between terminal and local segmental relaxation. Note also that while the observed relaxation time may be affected by other processes, such as constraint release [7], these do not alter the temperature dependence of linear chains.

When the polymer has long (entangled) branches, the temperature dependence of the rheology becomes more complicated. Since entangled branches suppress reptation, Graessley [8] suggested that terminal relaxation requires their retraction. The transient compact state associated with this retracted branch would give rise to a thermal barrier, $E^{*}$, with a magnitude dependent on the temperature coefficient of the chain coil size. Accordingly, the temperature dependence of the relaxation of branched polymers can then be described by an equation of the form [8,9]

$\ln \left[\frac{a_{\mathrm{Br}}(T)}{a_{\mathrm{lin}}(T)}\right] \propto E^{*} / \mathrm{RT}$

where $a(T)$ is the time-temperature shift factor. Eq. (4) implies a breakdown of the time-temperature superposition principle in the terminal zone for branched polymers.
According to Graessley et al. [8,9], the excess activation energy of branched polymers should be directly proportional to the branch length

$E^{*}=\Lambda M_{\mathrm{Br}} / M_{\mathrm{e}}$.

Since adoption of this transient compact structure requires an enhanced population of gauche (bent) rotamers, the proportionality constant $\Lambda$ should reflect the magnitude of the conformational energies. This leads to the prediction of a correspondence between $\Lambda$ and the temperature coefficient of the chain dimensions, $\kappa=\mathrm{d} \ln \left\langle r^{2}\right\rangle / \mathrm{d} T^{2}$ where $r$ is the end-to-end chain distance. Specifically, a negative $\kappa$ (expanded configuration energetically favored) is predicted for branched polymers showing thermorheological complexity in the terminal zone and an enhanced temperature dependence of viscosity. More recent work similarly invokes an energy barrier due to the configurations required for retraction of a branch, leading to the same qualitative predictions [10].

The best-known case is that of polyethylene. Consistent with its large and negative $\kappa$, polyethylene with long branches exhibits a breakdown of time-temperature superpositioning and an enhanced temperature coefficient for the viscosity $[11,12]$. However, it was noted early on that the model was only moderately successful in describing the available experimental results [9]. Hydrogenated polyisoprene and 1,2-polybutadiene have negative $\kappa$ values, and exhibit the predicted behavior. On the other hand, 1,4-polybutadiene has a positive $\kappa$, yet branching has a similar effect on its rheology.

1,4-polyisoprene (PI) has been the subject of various studies. Experiments covering only limited temperature ranges $\left(\leqslant 50^{\circ}\right)$ concluded that linear and star-branched PI had the same temperature dependence $[9,13]$. However, data obtained over a $120^{\circ}$ range of temperature showed clearly that there is an enhancement of the temperature dependence, accompanied by a breakdown of timetemperature superpositioning $[14,15]$. These results were supported by normal mode dielectric spectroscopy [16]. A value of $\Lambda=0.4 \mathrm{~kJ} / \mathrm{mol}$ was obtained. Although small, the fact that it is nonzero is at odds with the arm retraction model, since for polyisoprene $\kappa(=0.53[17])$ is positive and large. 
For polyisobutylene (PIB), $\kappa$ is close to zero $[17,18]$. Thus, the arm retraction models predict thermorheological simplicity and no difference in temperature dependence between branched and linear chains. This is exactly what was found both for stars [19] and for arborescent combs [20]. PIB is unusual in this regard; well-entangled branches have usually been found to enhance the temperature dependence of the rheological properties.

In this paper we present data concerning the temperature dependence of the terminal relaxation in polydimethylsiloxane (PDMS). PDMS has two features that make it well-suited for studying the effect of branching: it's glass temperature is unusually low, $T_{\mathrm{g}}=-123{ }^{\circ} \mathrm{C}$ and it is a saturated polymer with consequently good thermal stability. As a result, very large values of $T-T_{\mathrm{g}}$ can be achieved without degradation of the material. While this also implies that measurements might be made over a broad temperature range, in fact they are limited because of the propensity of PDMS to crystallize at lower temperatures.

\section{Experimental}

A linear PDMS, PDMS-1, was obtained from Scientific Polymer Products. It had a weight average molecular weight equal to $100 \mathrm{~kg} / \mathrm{mol}$, with a polydispersity of 1.16. A commercial PDMS, General Electric SE30 (designated PDMS-GE herein), was used as received. Additionally, a portion of it was mixed with an organic peroxide (Vanderbilt's Varox DCP-R), then reacted for 35 min at $175{ }^{\circ} \mathrm{C}$. As described below, this material was then fractionated prior to measurements. This sample is referred to herein as PDMS-GEx.

Dynamic mechanical spectra in the terminal zone were obtained on two Bohlin VOR rheometers, which had a combined torque range of from 10 to $2000 \mathrm{~g} \mathrm{~cm}$. A parallel plate geometry was employed, with sample radii of 12.5 or $20 \mathrm{~mm}$ and a $1.5 \mathrm{~mm}$ gap. The dynamic shear modulus was measured at angular frequencies in the range from 9 to $6 \times 10^{-5} \mathrm{rad} / \mathrm{s}$. Temperature control was $\pm 0.1^{\circ}$. The absence of degradation, particularly for results obtained at higher temperature, was confirmed by replicate measurements.

\section{Results}

\subsection{Material}

The fraction of polymer remaining soluble after reaction of the PDMS-GE with varying concentrations of dicumyl peroxide is shown in Fig. 1. The relationship between the weight fraction of soluble material, $W$, and the peroxide concentration, $c$, is given by the Charlesby-Pinner equation [21]

$W+\sqrt{W}=\left(G_{\mathrm{S}} / 2 G_{x}\right)+\left(2-G_{\mathrm{S}} / 2 G_{x}\right) \frac{c_{\text {gel }}}{c}$,

where $G_{\mathrm{S}}$ and $G_{x}$ are the rates of chain scission and crosslinking respectively, and $c_{\text {gel }}$ is the minimum level of peroxide yielding $W<1$. The peroxide crosslinking of the PDMS-GE does not yield the linearity suggested by Eq. (6). Nevertheless, by extrapolation we estimate that there are $\approx 0.5$ scissions per crosslinking event. As seen from Fig. $1, c_{\text {gel }}$ corresponds to less than $0.4 \%$ by weight of

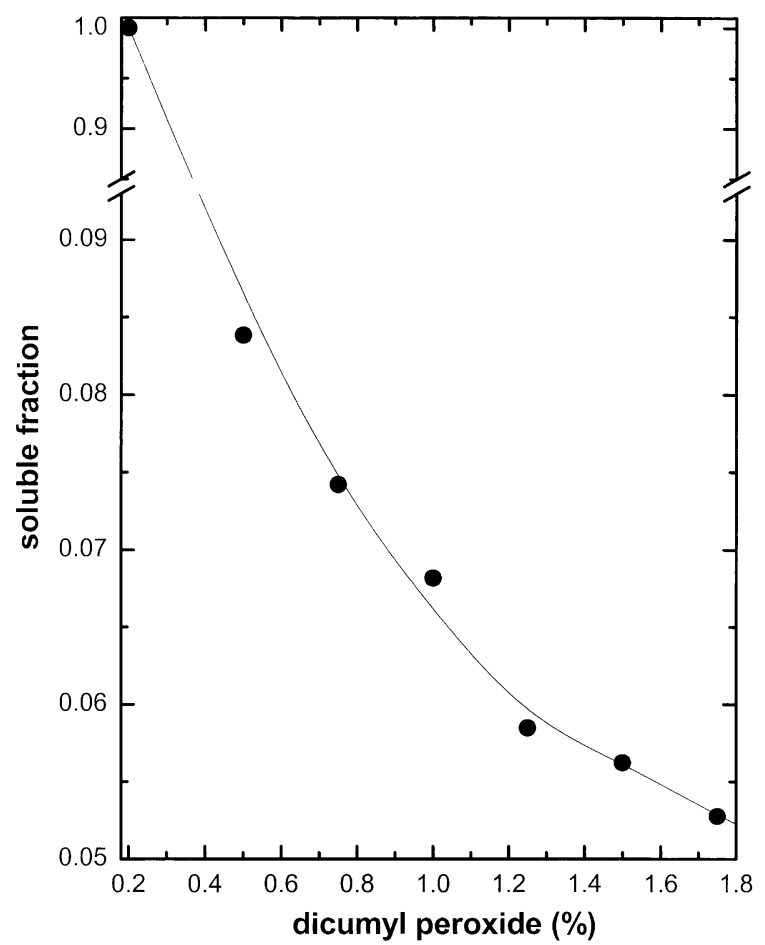

Fig. 1. Fraction of PDMS-GE remaining soluble after reaction with the indicated concentration (by weight) of dicumyl peroxide. 
the peroxide. At $0.2 \%$ peroxide level, the polymer remained completely soluble. This sample was fractionated using hexane and methanol. The initial precipitate (first cut $=$ least soluble fraction), designated PDMS-GEx, was used for dynamic mechanical testing.

\subsection{Dynamic mechanical results}

As illustrated in Fig. 2 by master curves of the phase angle measured at various temperatures, all samples exhibited thermorheological simplicity in the terminal zone. Also note that while PDMS-GE is approaching its zero-shear rate limiting behavior, the phase angle for PDMS-GEx is still much less than $90^{\circ}$; that is, terminal flow of the PDMSGEx occurs at substantially lower frequencies.

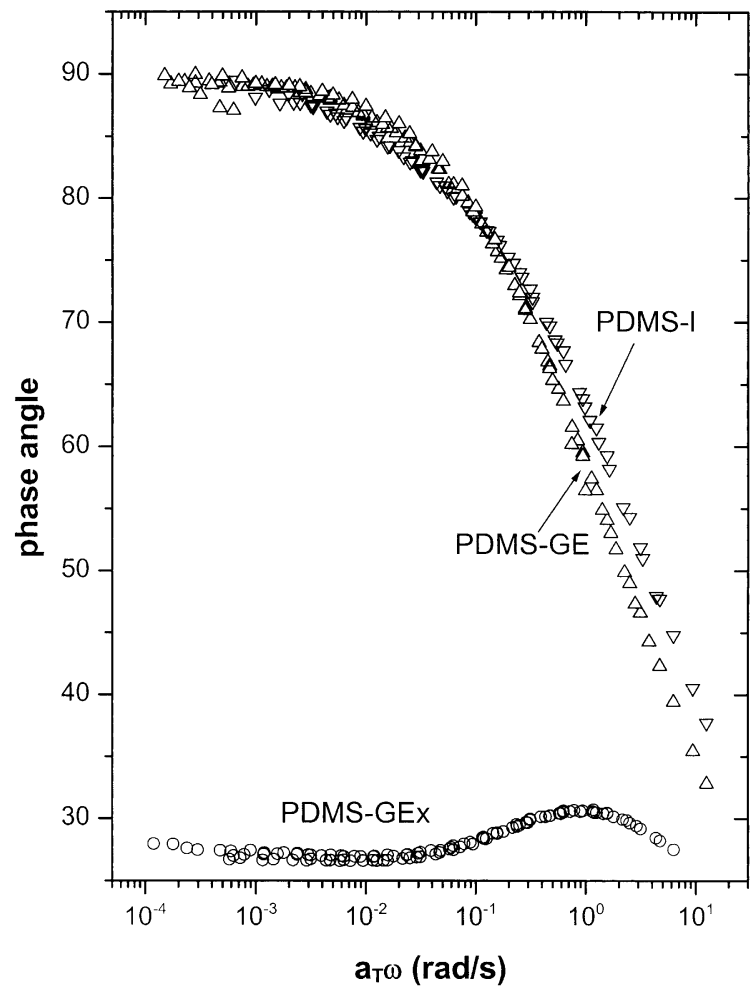

Fig. 2. Master curves of the phase angle for the linear PDMS $\left(\nabla\right.$ : data for 55,85 and $120^{\circ} \mathrm{C}$ ), PDMS-GE ( $\triangle$ : data for 10,70 , 105 and $135{ }^{\circ} \mathrm{C}$ ), and PDMS-GEx ( $\circ: 30,70,110$ and $130{ }^{\circ} \mathrm{C}$ ). The respective reference temperature for each sample is the lowest temperature. All materials exhibit thermorheological simplicity in the terminal zone.
The zero-shear rate limiting value of the dynamic viscosity for PDMS-1 was obtained over a temperature range from 10 to $140{ }^{\circ} \mathrm{C}$. At lower temperatures, the material crystallized during the measurements. These viscosity data (Fig. 3) are linear when plotted in Arrhenius form, yielding an activation energy equal to $E_{\mathrm{a}}=14.9 \mathrm{~kJ} / \mathrm{mol}$.

The viscosity of both PDMS-GE was large, and could only be measured over half a decade. The date, shown in Fig. 4, give $E_{\mathrm{a}}=15.9 \mathrm{~kJ} / \mathrm{mol}$. Since the terminal behavior is thermorheologically simple (Fig. 2), we can extend the temperature range by plotting shift factors. Specifically, we determine the frequency of the maximum in the loss modulus, the inverse of which defines a characteristic time, $\tau_{\mathrm{p}}$. This is plotted in Fig. 5. The data are linear, and from the slope we obtain $E_{\mathrm{a}}=17.4 \mathrm{~kJ} /$ mol.

Thus, the activation energy for the terminal relaxation time is $1.5 \mathrm{~kJ} / \mathrm{mol}$ larger than $E_{\mathrm{a}}$ for the viscosity. The theoretical relationship between

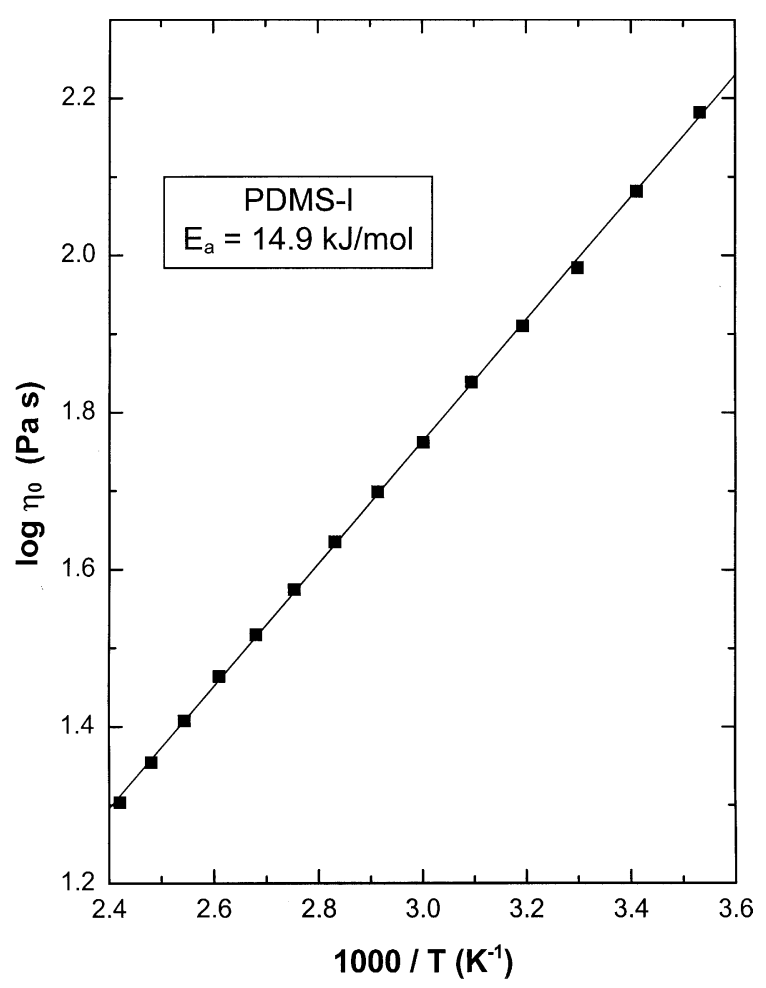

Fig. 3. Terminal (zero-shear) viscosity of the linear PDMS. 


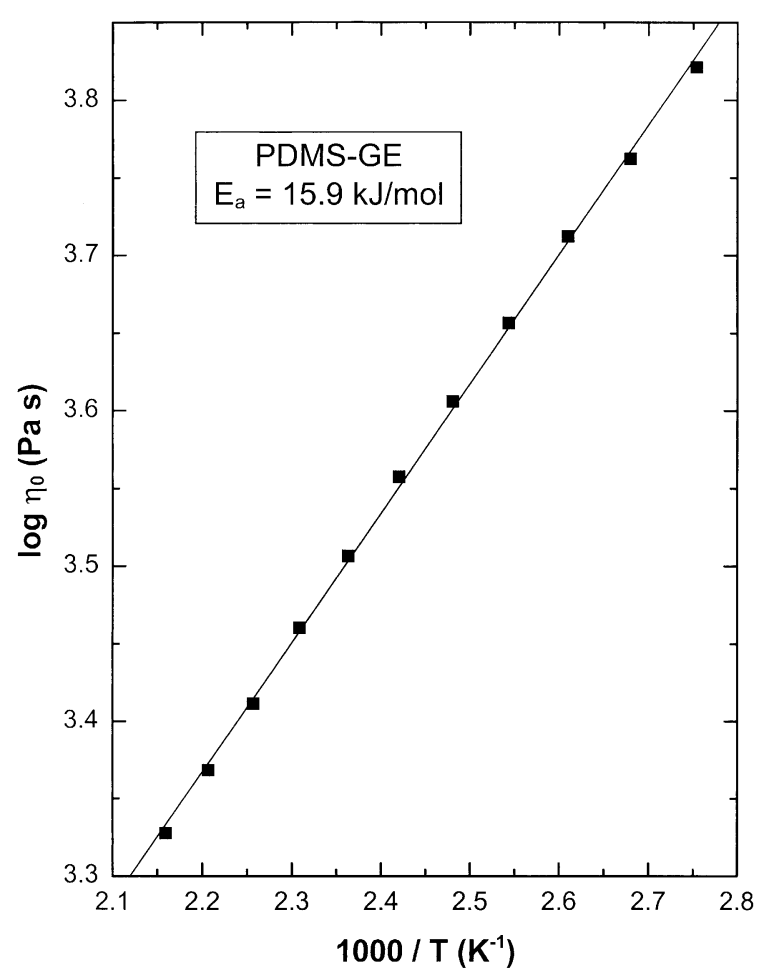

Fig. 4. Terminal viscosity of the commercial PDMS.

these two quantities is given by Eq. (3). The temperature dependence of $\tau_{\mathrm{p}}$ is expected to exceed that of $\eta_{0}$ by an amount equal to the variation with temperature of the ('vertical') shift factors, $b_{T}$, describing the temperature dependence of the modulus. Although various relations have been proposed for these shift factors [1,22], $b_{T}$ were determined empirically herein. The results are shown in Fig. 6 in Arrhenius form, indicating an apparent activation energy equal to $1.6 \mathrm{~kJ} / \mathrm{mol}$. This is in good agreement with the difference between $E_{\mathrm{a}}$ obtained for the viscosity (Fig. 4) and the relaxation times (Fig. 5).

For the very viscous PDMS-GEx, we eschewed measurement of $\eta_{0}$. The temperature dependence of this sample was assessed from $\tau_{\mathrm{p}}$, and from time-temperature shift factors measured in the terminal zone. Since crystallization was suppressed in the chemically reacted polymer, measurements could be obtained at temperatures down to $-40{ }^{\circ} \mathrm{C}$. As seen in Fig. 7, the Arrhenius plot for

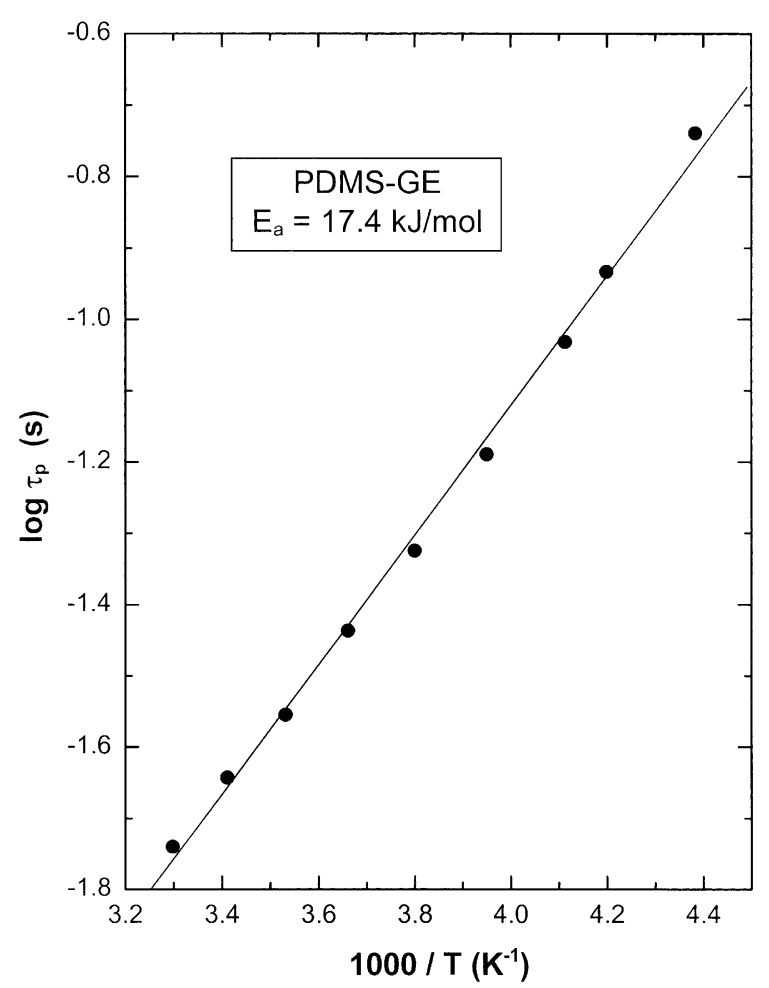

Fig. 5. Inverse of the frequency of the maximum in the loss modulus for the commercial PDMS.

PDMS-GEx is linear, yielding an activation energy, $E_{\mathrm{a}}=17.0 \mathrm{~kJ} / \mathrm{mol}$.

\section{Discussion}

Previous assessments [9,14-16] of the effect of branching on the temperature dependence relied on the assumption that the mechanism to alleviate constraints from entangled branches was a thermally activated process [8]. This means that an Arrhenius plot of terminal relaxation times or viscosities for a branched polymer, normalized by that of the corresponding linear species, would be linear (Eq. (4)). The slope yields an activation energy, reflecting the degree to which branching enhances the temperature coefficient of the viscosity. The advantage of studying PDMS is that linear Arrhenius plots are obtained directly (viz. Figs. 3-5 and 7). This is a consequence of the low glass temperature of PDMS, which, in 


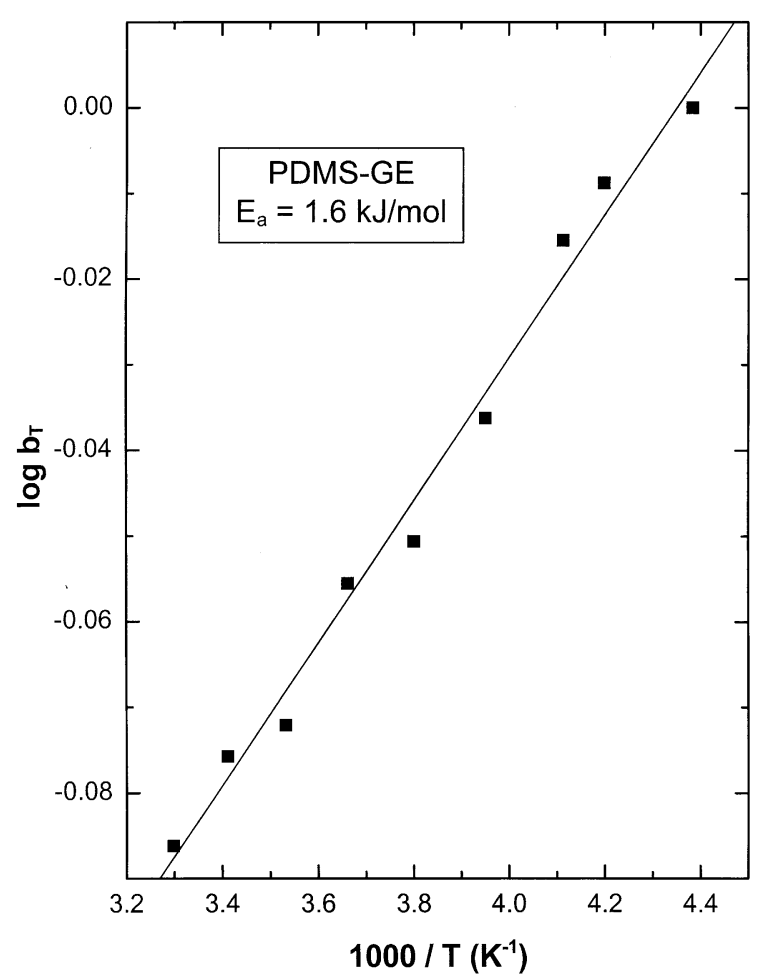

Fig. 6. Modulus (vertical) shift factors for the terminal zone of the commercial PDMS.

combination with its good thermal stability, allowed measurements at temperatures more than $270^{\circ}$ above $T_{\mathrm{g}}$. The consequence is linear Arrhenius plots of the viscosity and shift factors, obviating the need to normalize the branched polymer results by data for the linear PDMS. Accordingly, analysis of the enhancement of the temperature dependence herein is model-independent.

The activation energy for the viscosity of PDMS-GE is $6 \%$ larger than $E_{\mathrm{a}}$ determined for $\eta_{0}$ of PDMS-1, a difference that is close to the experimental error. Plazek et al. [23] reported a flow activation energy of $15.3 \mathrm{~kJ} / \mathrm{mol}$, for a PDMS the authors assumed to be linear [24]. This value is intermediate between the $E_{\mathrm{a}}$ in of our two samples. From these results, we can conclude that either (i) the commercial PDMS lacks long-chain branching, or (ii) branching does not significantly affect the temperature dependence of the rheology of PDMS.

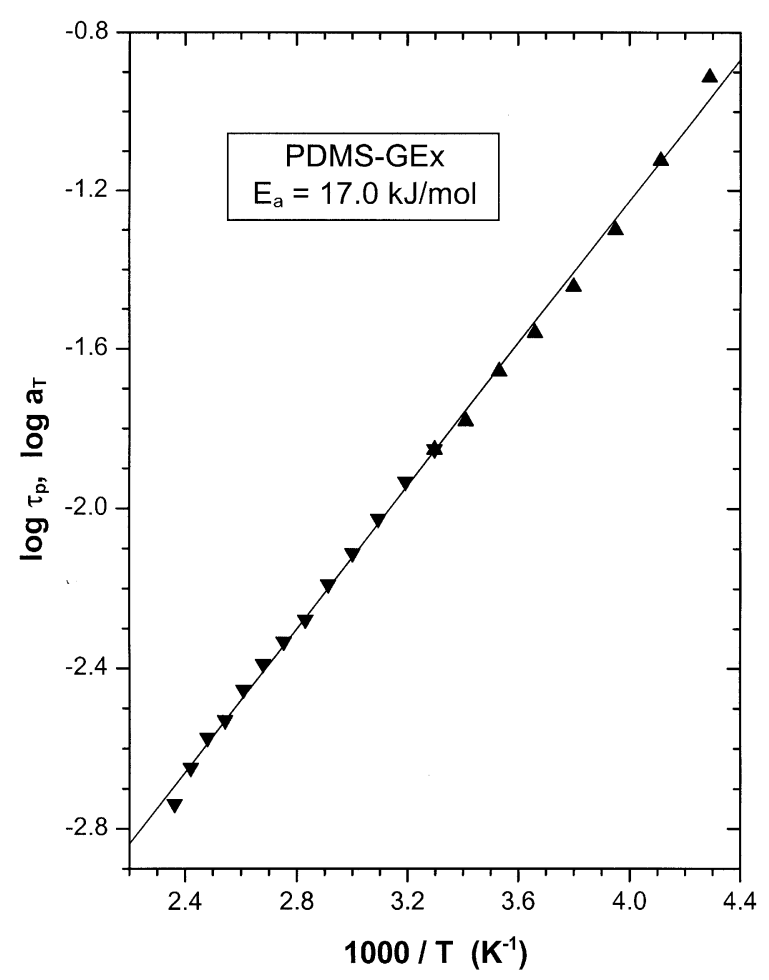

Fig. 7. Inverse of the frequency of the loss modulus maximum $(\boldsymbol{\Delta})$ and the terminal zone shift factors $(\boldsymbol{\nabla})$ for the commercial PDMS after reaction with peroxide and fractionation.

As seen in Figs. 5 and 7, the respective activation energies for PDMS-GE and PDMS-GEx are equal, within the experimental error. They are also consistent with the temperature coefficient of the viscosity for PDMS-1, when adjusted using Eq. (3). The chemically-reacted PDMS is certainly branched. Moreover, these branches are an appreciable fraction of the primary chain length, and thus well entangled. Thus, we can conclude that branching does not affect the temperature dependence.

More usually, enhancement of the temperature dependence is observed for polymers with wellentangled branches $[9,11,12,14-16]$, and it is accompanied by a breakdown of time-temperature superpositioning in the terminal zone. In this regard, the thermorheological simplicity seen in Fig. 2 is consistent with a lack of such enhancement in PDMS. Similar results were reported for PIB $[19,20]$. This correspondence between the effects of branching on the temperature dependence and 
on thermorheological complexity is predicted by models [8-10]. The idea is that retraction of entangled branches is necessary for terminal relaxation in branched polymers. This retraction mechanism is thermally activated, and should be affected differently by temperature changes than other terminal relaxation processes (e.g., reptation). However, at temperatures sufficiently high that the terminal relaxation sans arm retraction exhibits Arrhenius behavior, no breakdown is expected. Rather, a single activation energy would be observed, reflecting the cumulative temperature dependence of both processes. Thus, the thermorheological simplicity observed herein is inevitable, regardless of the relative magnitudes of $E_{\mathrm{a}}$ for the branched and linear PDMS.

Rheological models for branched polymers also predict a correspondence between the enhancement in the temperature dependence and the temperature coefficient of the polymer's chain dimensions. Specifically, the prediction is that an increased temperature dependence will only arise in polymers for which $\kappa$ negative [8-10]. For PDMS, $\kappa=5.9 \times 10^{-4} \pm 1.4 \times 10^{-4}$ [17]. The sign of $\kappa$ is corroborated by mechanical results on PDMS networks: The energetic component of the rubbery elastic stress, whose fractional contribution is equal to $\kappa$ times $T$, is also positive [17].

Although the present results are consistent with theory, unresolved issues remain. PI is similar to PDMS, in that $\kappa$ is positive. In fact, within the reported uncertainties, $\kappa$ for PI is numerically equal to the value for PDMS [17]. Yet long chain branching enhances the temperature coefficient of the terminal relaxation for PI, and gives rise to thermorheological complexity [14-16].

\section{Summary}

Terminal relaxation measurements were carried out on linear and branched PDMS over temperatures for which Arrhenius behavior obtained. Both the linear and branched samples were thermorheological simple. The temperature dependence of the rheology was equivalent for branched and linear PDMS. Since the temperature coefficient of the chain dimensions for PDMS is positive, this invariance of the temperature dependence to branching is consistent with models for the rheology of branched polymers, invoking an arm retraction mechanism for terminal relaxation.

\section{Acknowledgements}

This work was supported by the Office of Naval Research. Stimulating discussions with D.J. Plazek are gratefully acknowledged.

\section{References}

[1] J.D. Ferry, Viscoelastic Properties of Polymers, Wiley, New York, 1980.

[2] K.L. Ngai, D.J. Plazek, Rubber Chem. Technol. 68 (1995) 376.

[3] P.G. Santangelo, K.L. Ngai, C.M. Roland, Macromolecules 26 (1993) 2682.

[4] M. Doi, S.F. Edwards, Theory of Polymer Dynamics, Clarendon, Oxford, 1986.

[5] D.J. Plazek, I.-C. Chay, K.L. Ngai, C.M. Roland, Macromolecules 28 (1995) 6432.

[6] P.G. Santangelo, C.M. Roland, Macromolecules 31 (1998) 3715.

[7] D.S. Pearson, D.S. Rubber, Chem. Technol. 60 (1987) 439.

[8] W.W. Graessley, Macromolecules 15 (1982) 1164.

[9] J.M. Carella, J.T. Gotro, W.W. Graessley, Macromolecules 19 (1986) 659.

[10] A.J. Levine, S.T. Milner, Macromolecules 31 (1998) 8623.

[11] G.C. Berry, T.G. Fox, Adv. Polym. Sci 5 (1968) 261.

[12] W.W. Graessley, Acct. Chem. Res. 10 (1977) 332.

[13] S.T. Milner, T.C.B. McLeish, Macromolecules 31 (1998) 7479.

[14] C.A. Bero, C.M. Roland, Macromolecules 29 (1996) 1562.

[15] P.G. Santangelo, C.M. Roland, J. Non-Cryst. Solids 235 (1998) 709.

[16] C.M. Roland, C.A. Bero, Macromolecules 29 (1996) 7521.

[17] B. Erman, J.E. Mark, Structures and Properties of Rubberlike Networks, Oxford University, New York, 1997.

[18] U.W. Suter, E. Saiz, P.J. Flory, Macromolecules 16 (1983) 1317.

[19] P.G. Santangelo, C.M. Roland, J.E. Puskas, Macromolecules 32 (1999) 1972.

[20] C.G. Robertson, C.M. Roland, C. Paulo, J.E. Puskas, J. Rheology 45 (2001) 759.

[21] A. Charlesby, S.H. Pinner, Proc. R. Soc. London 249 (1959) 367.

[22] W.W. Graessley, S.F. Edwards, Polymer 22 (1981) 1329.

[23] D.J. Plazek, W. Dannhauser, J.D. Ferry, J. Coll. Sci. 16 (1961) 101.

[24] D.J. Plazek, private communication. 